\title{
Lack of plakoglobin impairs integrity and wound healing in corneal epithelium in mice
}

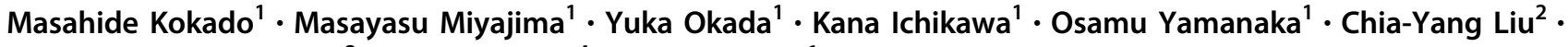 \\ Winston Whei-Yang $\mathrm{Kao}^{3} \cdot$ Weinian Shou ${ }^{4} \cdot$ Shizuya Saika ${ }^{1}$
}

Received: 28 February 2018 / Revised: 28 March 2018 / Accepted: 31 March 2018 / Published online: 25 May 2018

(c) United States \& Canadian Academy of Pathology 2018

\begin{abstract}
We generated cornea-specific plakoglobin (Jup; junctional plakoglobin) knockout mice in order to investigate the function of plakoglobin on the maintenance of the homeostasis of corneal epithelium in mice. Cornea epithelium-specific conditional knockouts $\left(J_{u p}{ }^{C E \Delta / C E \Delta}\right)(\mathrm{cKO})$ were obtained by breeding keratin12-Cre (Krt12-Cre) mice to Jup-floxed (Jup ${ }^{f f f}$ mice. Light and transmission electron microscopic and immunohistochemical analyses were carried out to determine consequence of the loss of plakoglobin on maintaining corneal epithelium integrity under mechanical stress, e.g., brushing and wound healing. Immunohistochemistry analysis demonstrated that, although Jup ablation did not affect BrdU incorporation, basal cell-like cells labeled for keratin 14 were ectopically present in the supra-basal layer in mutant corneal epithelium, suggestive of altered cell differentiation. Plakoglobin-deficient epithelium exhibits increased fragility against mechanical intervention when compared to wild-type controls under identical treatment. Closure of an epithelial defect was significantly delayed in $J u p^{C E \Delta / C E \Delta}$ epithelium. Our findings indicate that the lack of plakoglobin significantly affects corneal epithelium differentiation, as well as its structural integrity. Plakoglobin is essential to the maintenance of the structure of the corneal epithelium and its wound healing.
\end{abstract}

\section{Introduction}

Cornea locates to the surface of the anterior eye segment, facing outer environment. It is covered with non-keratinizing epithelium associated with a smooth basement membrane, and is a non-vascularized tissue without hair follicle. Homeostasis of the corneal epithelium is critical in exerting a barrier function to noxious external stimuli, i.e., scratches, injury, dryness, chemicals and pathogens. Steady and rapid resurface of corneal epithelial defects is essential to the prevention of invasion of pathogens as well as of further

Shizuya Saika

shizuya@wakayama-med.ac.jp

1 Department of Ophthalmology, Wakayama Medical University School of Medicine, Wakayama, Japan

2 School of Optometry, Indiana University, Bloomington, USA

3 Department of Ophthalmology, University of Cincinnati, Cincinnati, USA

4 Herman B Wells Center for Pediatric Research, Indiana University School of Medicine, Indianapolis, IN, USA damage of the underlying stroma. Intercellular junctions between neighboring epithelial cells, i.e., desmosomes, adherence junctions, and tight junctions play significant roles to maintain the barrier function [1-3]. Plakoglobin (junctional plakoglobin, Jup, also called $\gamma$-catenin) is a member of the catenin family and is a common component to both adherens junctions and desmosomes [4]. Both plakoglobin and $\beta$-catenin share the structural role in the adherence junction, while plakoglobin in the desmosome can not be replaced by $\beta$-catenin [5]. Loss-of-function mutation of plakoglobin causes Naxos disease, an autosomal recessive disease characterized by palmoplantar keratoderma and cardiomyopathy [6, 7]. Palmoplantar keratoderma exhibits thick hyperkeratosis over the palms and soles. Wnt signal abnormality was reported in the affected epidermis probably due to consumption of $\beta$-catenin to compensate the loss-of-function of plakoglobin [8].

Global expression of nonsense mutation of plakoglobin gene results in embryonic lethality with congenital epidermolysis in mice, much different from human phenotype of Naxos disease [9]. We previously generated a mouse line with keratinocyte-specific inactivation of plakoglobin, which manifested clinical features resembling human palmoplantar 
Table 1 Targets of Immunohistochemistry and the Sources of the Antibodies
Keratin14

Plakoglobin

$\beta$-Catenin

Epiplakin (diluted 1:100 in PBS; abcam, Cambridge, England)

(diluted 1:10; Progen Biotechnik, Heidelberg,Germany)

(diluted 1:100; BD Transduction Laboratories, San Jose, CA)

(diluted 1:100; donated by Dr. S. Fujiwara, J Dermatol 33; 518, 2006) keratodermia e.g., over-cornification and thickening of the epidermis with inflammation [10]. Ultrastructural examination shows cell-cell contact was severely impaired. The structural study failed to validate the effects of inactivation of plakoglobin on epithelial wound healing, albeit the structure and behaviors of epidermis that lacks plakoglobin strongly suggests the alteration of wound healing-related cell behaviors of a keratinocyte.

Corneal epithelium is quite suitable for the investigation of the role of a specific molecule in homeostasis of stratified epithelium and wound healing reaction of an epithelial tissue. There is a possibility by which lacking one specific cell adhesive molecule abnormality impairs the homeostasis of the corneal epithelium [11]. We previously reported that corneal epithelium-specific deletion of epiplakin impairs intra-epithelial differentiation and integrity of corneal epithelium, but accelerates epithelial wound healing [11]. However, the role of the plakoglobin in epithelial repair in cornea has not been well examined in keratinocyte-specific plakoglobin knockout mice, which is largely due to that previously used keratin 14-driven Cre recombinase could affect epithelial health of not only eye, but also eyelids, by deleting plakoglobin in epithelium of total ocular surface (cornea, limbus, and conjunctiva) as well as in eyelid skin [10]. In the current study we investigate the role of plakoglobin in the maintenance of structural integrity and in wound healing of corneal epithelium in mice by using corneal epithelium-specific keratin12 promoter-driven Cre recombinase to ablate floxed junctional plakoglobin ( Jup) gene.(cKO)

\section{Materials and methods}

\section{Mice}

$J u p^{f l f}$ mice and keratin 12 (Krt12)-Cre knock-in (Krt12-Cre) mice were both described previously [12, 13]. All studies involving the use of mice were approved by the DNA Recombination study committee and Animal Care and Use Committee both of Wakayama Medical University and conducted in compliance with the Association for Research in Vision and Ophthalmology Statement for the Use of Animals in Ophthalmic and Vision Research. Jup ${ }^{f l f}$ mice and $\mathrm{Krt} 12^{\mathrm{Cre}}$ knock-in mice were cross-bred to obtain $\mathrm{Krt} 12^{\mathrm{Cre} / \mathrm{Cre}} / \mathrm{Jup}^{\mathrm{f} / \mathrm{f}}\left(\mathrm{Jup}^{\mathrm{CEACE \Delta}}\right)$ in which plakoglobin gene is specifically ablated in corneal epithelium. We successfully obtained two Jup ${ }^{C E \Delta / C E \Delta}$ mice out of 202 bitransgenic mice because both $\mathrm{Krt} 12^{\mathrm{Cre}}$ and $\mathrm{Jup}^{f}$ alleles are located in mouse chromosome 11 about $0.55 \mathrm{cM}$ apart. Then the Jup ${ }^{C E \Delta C E \Delta}$ colony was expanded for experiments described herein.

\section{Histology and ultrastructure of corneal epithelium in the absence of plakoglobin}

The structure of corneal epithelium of a wild-type (WT) and a mutant mouse was examined by light microscopy of routine hematoxylin and eosin (HE)-stained paraffin sections and transmission electron microscopy as previously reported [14, 15]. The arrangement of epithelial cells in the corneal epithelium was further investigated as follows: to determine the longitudinal basal corneal epithelial cell density, the number of DAPI-stained nuclei in $600 \mu \mathrm{m}$ along the basement membrane and total number of nuclei in stratified layers of the same region were counted from seven independent specimens.

\section{Characterization of corneal epithelium by using immunohistochemistry}

Immunohistochemistry was carried out to characterize the nature of the epithelial cell of the cornea in the absence of plakoglobin. The antibodies listed in Table 1 were used.

\section{Examination of fragility of the corneal epithelium}

Our previous method of mechanical brushing of the epithelium was employed herein to examine if lacking plakoglobin affects the integrity of the corneal epithelium in mice [11]. The corneal surface of adult WT and mutant mice, under general anesthesia, was gently brushed (ten times) with a dry surgical micro-sponge as previously reported $[11,16]$. Mice were killed and the eye was enucleated and embedded in Epon mixture as previously reported [16]. Ultrathin sections were cut and observed under routine transmission electron microscopy after electron staining.

\section{Epithelial wound healing in cornea}

Closure of injuries in corneal epithelium was examined to elucidate the role of plakoglobin on epithelium repair as previously reported [14]. A circle defect (2 $\mathrm{mm}$ in diameter) was produced in the corneal epithelium of WT mice $(n=5)$ 
and $J u p^{C E \Delta / C E \Delta}$ mice $(n=5)$. The size of the remaining injury was evaluated with fluorescein staining at every $6 \mathrm{~h}$ until $42 \mathrm{~h}$ post-wounding.

\section{Cell proliferation assay}

The incidence of proliferating cells in corneal epithelium was evaluated by using immuno-detection of systemically administered bromo-deoxyuridine (BrdU) in corneal epithelium as previously reported [17]. The examination was performed in a healthy, uninjured, cornea and in the cornea 18 and $48 \mathrm{~h}$ post epithelial debridement $(n=22$ in each experimental condition).

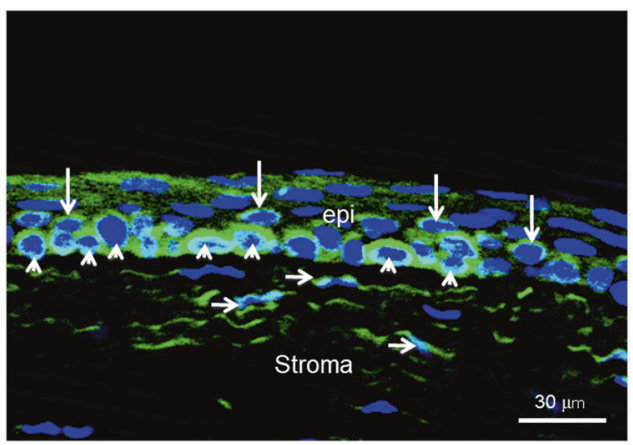

Fig. 1 Immunohistochemical detection of plakoglobin in the corneal epithelium of a wild type (WT) mouse. Immunohistochemistry detected plakoglobin protein in mainly cell-cell junction zone and cytoplasm of the basal cells (arrowheads) and of some of the suprabasal cells (long arrows) of uninjured epithelium of a WT cornea. Keratocytes (short arrows) are also labeled for plakoglobin protein. Epi epithelium. Bar, $30 \mu \mathrm{m}$

Fig. 2 Histology and ultrastructure of corneal epithelium in the absence of plakoglobin. a Light microscopy by hematoxylin and eosin (HE) staining showed that the normal corneal epithelium of a wild type (WT) mouse consisted of basal cells, wing cells and superficial cells. $\mathbf{b}$ In the absence of plakoglobin epithelium, HE histology did not reveal marked abnormalities under light microscopy. c, $\mathbf{d}$ Low magnification transmission electron microscopy showed differentiation process from basal cells (white asterisks) toward suprabasal (stars) and superficial cells (black asterisks) both in WT and in the absence of plakoglobin. Epi epithelium. Bar, $50 \mu \mathrm{m}(\mathbf{a}, \mathbf{b}) ; 10 \mu \mathrm{m}(\mathbf{c}, \mathbf{d})$

\section{Results}

\section{Protein expression pattern of plakoglobin in normal mouse corneal epithelium}

Plakoglobin protein was clearly detected in the basal cells of WT corneal epithelium. In the suprabasal and superficial cells less slightly weaker green fluorescence was observed in the cytoplasm (Fig. 1). Keratocytes in the stroma was also labeled for plakoglobin (Fig. 1).

\section{Histology and ultrastructure of corneal epithelium in the absence of plakoglobin}

HE staining did not reveal marked abnormalities in the corneal epithelium between WT and cKO mice (Fig. 2a, b). Low magnification transmission electron microscopy showed gradual differentiation process from basal cells toward suprabasal and superficial cells in both genotypes of mice (Fig. 2c, d). However, higher magnification observation revealed reduction and less prominent formation of intercellular adhesion complex associated with vacuole-like spaces between neighboring cells in the superficial layer of mutant Jup ${ }^{C E \Delta C E \Delta}$ mice in comparison to that of WT mice (Fig. 3a, b). Such abnormality was not observed among the basal cells even in the cKO mice (Fig. 3c, d). High-magnification pictures show well-formed the basement membrane and hemidesmosome was also noted in both genotype of mice (Fig. 3e, f).
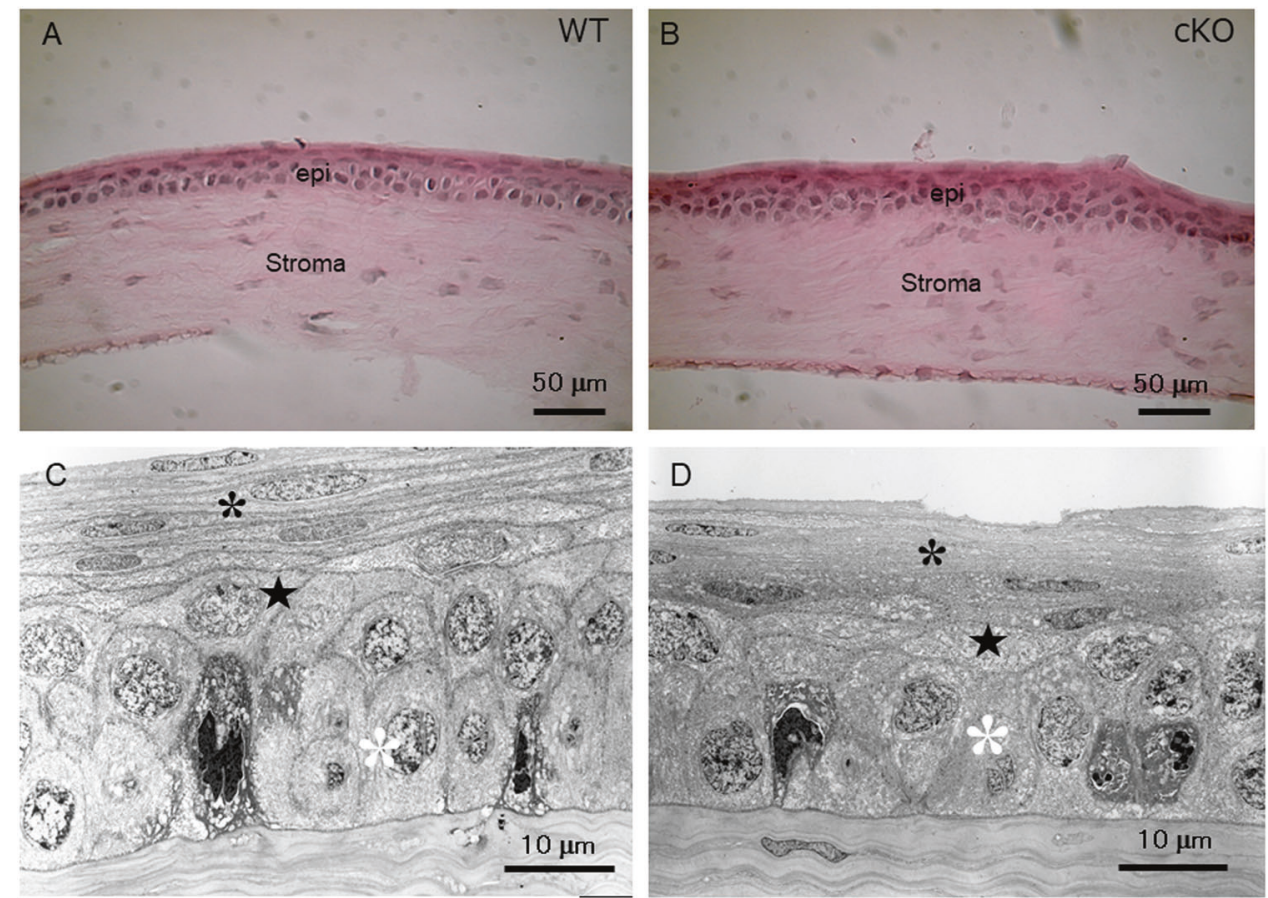
Fig. 3 Higher magnification observation of corneal epithelium in the presence or absence of plakoglobin. Higher magnification observation revealed attenuation of the formation of cell-cell adhesion with intercellular dissociation (arrows) among superficial cells in the absence of plakoglobin (b), while cell-cell adhesion is compact in a WT cornea (a). Adhesion or connection between neighboring cells seems to be normal in the presence (c) or absence (d) of plakoglobin. High-magnification pictures show well-formed the basement membrane and hemidesmosome was also noted in both genotype of mice (e, f). Bar, $2 \mu \mathrm{m}(\mathbf{a}, \mathbf{b})$; $3 \mu \mathrm{m}(\mathbf{c}, \mathbf{d})$
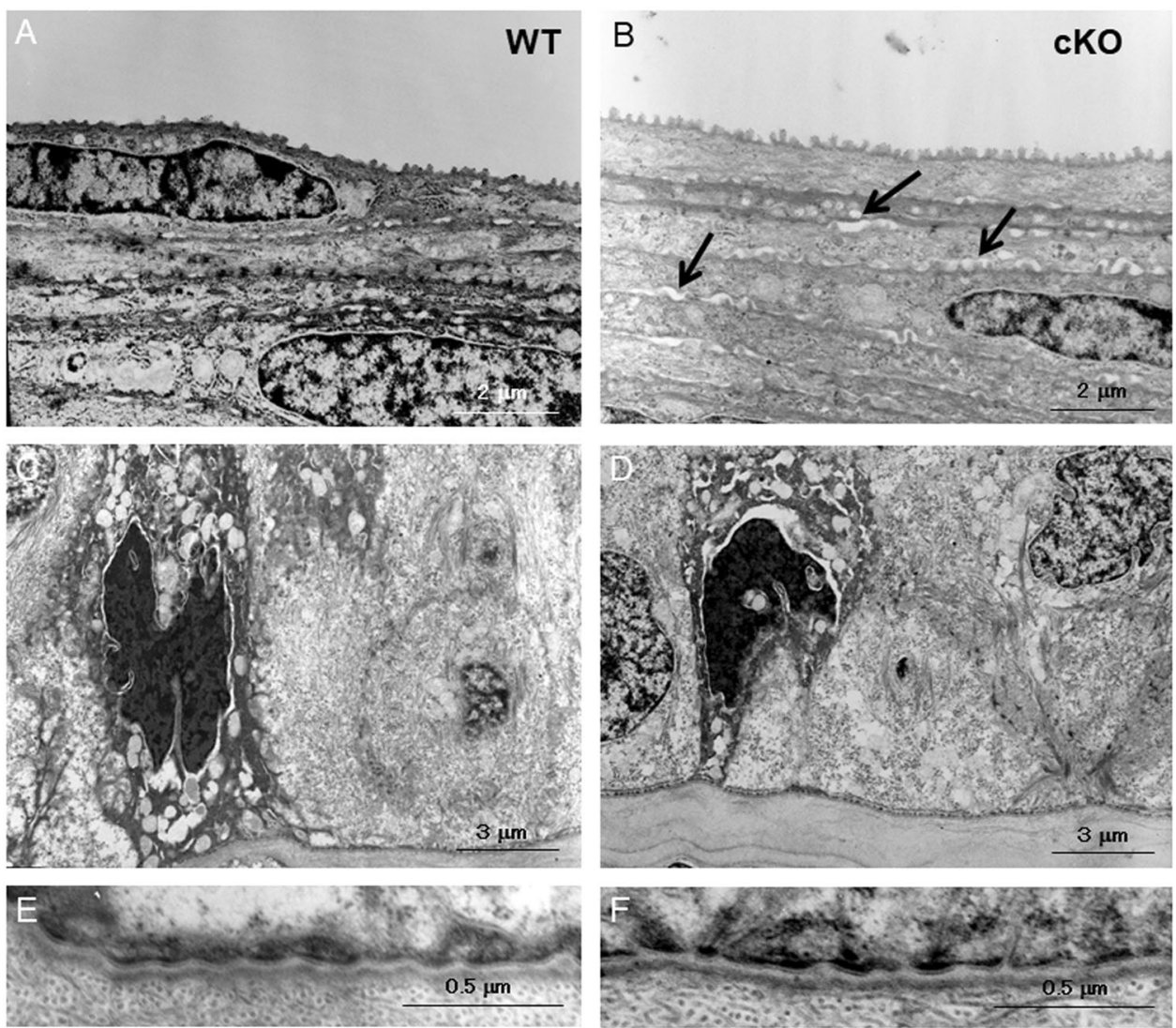

a

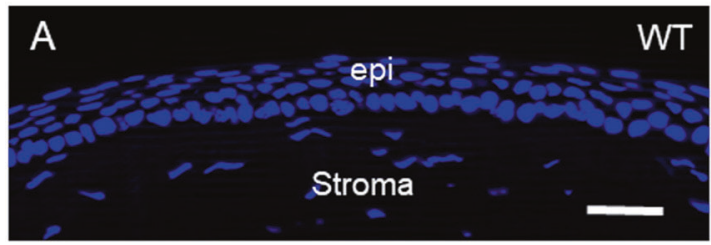

b

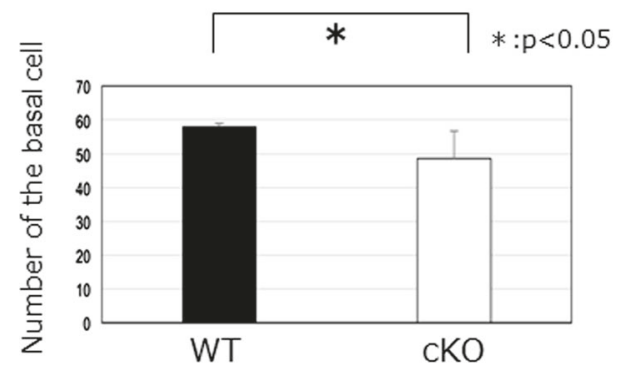

Fig. 4 The cell density in the corneal epithelium evaluated by DAPI nuclear staining. a DAPI staining clearly shows the spatial population of epithelial cells. Epi epithelium. Bar, $30 \mu \mathrm{m}$. b The number of basal cells in a specific area ( $600 \mu \mathrm{m}$ in length of the basement membrane)

\section{The cell density in the corneal epithelium was evaluated by DAPI nuclear staining}

Although ultrastructural observation did not suggest the impairment of cell-cell adhesion in basal cells in a cKO
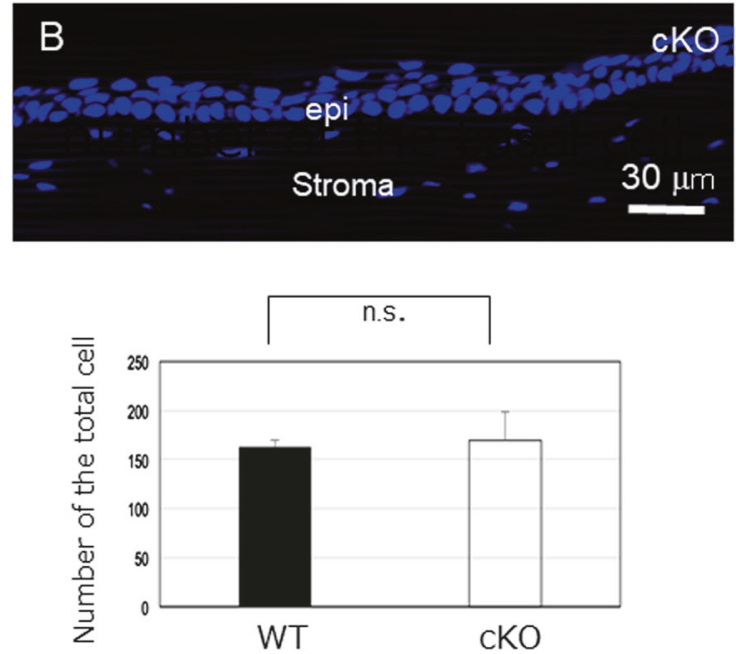

decreased in the absence of plakoglobin. However, the total number of the nuclei of any epithelial cells in a specific area $(600 \mu \mathrm{m}$ in length of the basement membrane) was unchanged by the loss of plakoglobin

epithelium, the loss of plakoglobin might attenuate tightness of cell-cell contact. To examine the spatial population of epithelial cells we counted the numbers of basal cells overlaying the basement membrane and the total numbers of cells within the same region by DAPI-nuclear staining (Fig. 4a). 

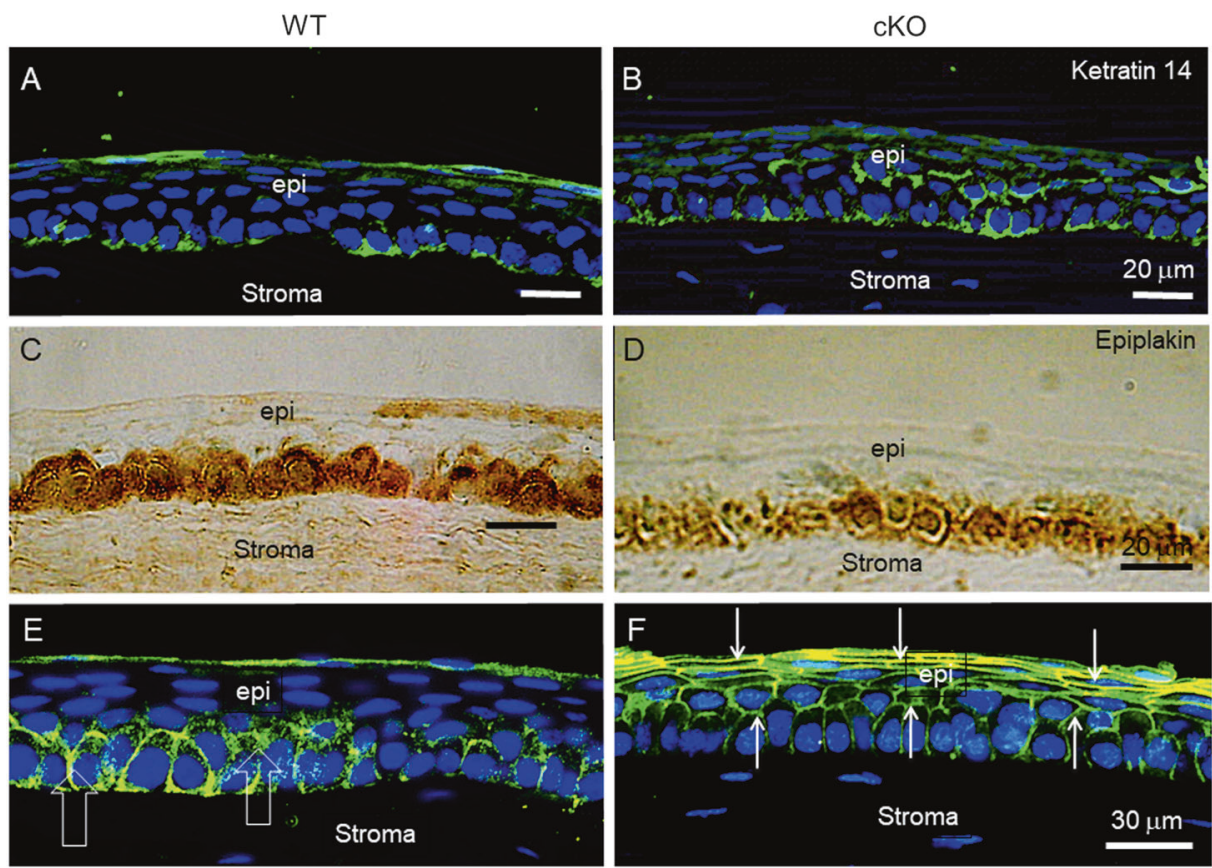

Fig. 5 Characterization of wild-type (WT) or mutant corneal epithelium by using immunohistochemistry for keratin 14 or epiplakin. Keratin 14, a basal cell-type keratin, was detected in the basal cells in a WT cornea. a On the other hand, keratin 14-immunoreactivity was observed in epithelial cells in the basal and suprabasal layers. b Epiplakin, a desmosome-associated protein component for the maintenance of cell fragility, was expressed in the basal cells of a WT

The results showed that the number of basal cells in central cornea $(600 \mu \mathrm{m}$ in length of the basement membrane) decreased in the absence of plakoglobin (Fig. 4b). However, the total number of the nuclei of any epithelial cells in a specific area $(600 \mu \mathrm{m}$ in length of the basement membrane) was unchanged by the loss of plakoglobin in mutants (Fig. 4b). This finding suggests that decrease in the basal cells is not due to the reduction of supplying daughter cells.

\section{Characterization of corneal epithelium by using immunohistochemistry}

Keratin 14, a basal cell-type keratin, was detected in the basal cells in the WT cornea (Fig. 5a). On the other hand, marked keratin 14-immunoreactivity was observed in epithelial cells in the basal and suprabasal layers of the mutant corneas (Fig. 5b). Epiplakin, a desmosome-associated protein component for the maintenance of cell fragility, was expressed in the basal cells of the WT corneal epithelium, while the intensity of immunostaining was reduced by the loss of plakoglobin (Fig. 5c, d).

Localization of $\beta$-catenin was dramatically altered by the loss of plakoglobin in corneal epithelium. $\beta$-catenin was detected in the cytoplasm of the basal cells of the WT corneal epithelium. Its cytoplasmic localization was

corneal epithelium (c), while the intensity of immunostaining was reduced by the loss of plakoglobin (d). $\beta$-catenin was detected in the nuclei of superficial cells of a WT corneal epithelium (open arrows) (e). $\beta$-catenin was observed between suprabasal cells and superficial cells and in the area between basal cells and basement membrane in a mutant epithelium (thin arrows) (f). Epi epithelium. Bar, $20 \mu \mathrm{m}$ $(\mathbf{a}-\mathbf{d}), 30 \mu \mathrm{m}(\mathbf{e}, \mathbf{f})$

diminished by the loss of plakoglobin, while $\beta$-catenin was markedly condensed in cell-cell-border of epithelial cells throughout the layers of the cKO mouse (Fig. 5e, f).

\section{Examination of integrity/fragility of the corneal epithelium}

Plakoglobin is a component of desmosome. Although the loss of plakoglobin in desmosomes could be compensated by $\beta$-catenin, its effect on epithelial integrity was to be determined. Moreover, current immunohistochemistry showed that lacking plakoglobin gene downregulates the expression of epiplakin, that is essential to the maintenance of epithelial integrity [11].

Ultrastructural observation showed that gentle brushing with a surgical micro-sponge did not damage the structure of the corneal epithelium in WT mice (Fig. 6a, b). On the other hand, the cells in the superficial layer were totally removed by the treatment in a mutant epithelium (Fig. 6d, e). The suprabasal cells and basal cells seemed to be maintained following the brushing treatment in the mutant cornea in a cKO epithelium (Fig. 6f), suggesting the superficial cells were removed at the cell-cell border between suprabasal cells. Basement membrane looked unaffected by the treatment (Fig. 6c, f). 

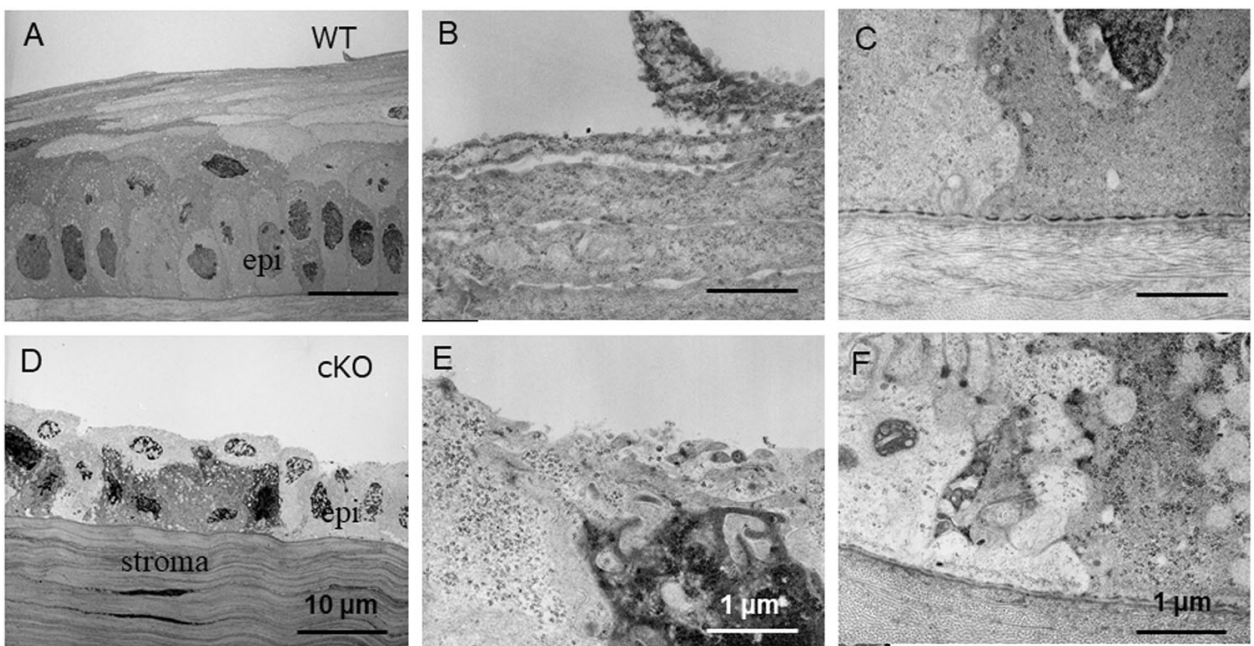

Fig. 6 Examination of fragility of wild-type (WT) or mutant corneal epithelium. Ultrastructural observation showed that gentle brushing with a surgical micro-sponge did not damage the structure of the corneal epithelium in a WT mouse (a). A higher magnification shows that the superficial layer is partially removed by the treatment (b). Basement membrane is intact in the WT tissue after treatment (c). The cells in the superficial layer were totally removed by the treatment in a mutant epithelium $(\mathbf{d})$. A higher magnification indicates that the The suprabasal cells and basal cells seemed to be maintained following the brushing treatment in a mutant cornea, suggesting the superficial cells were removed at the cell-cell border between suprabasal cells (e). Basement membrane looked unaffected by the treatment even in a mutant epithelium (f). Bar, $10 \mu \mathrm{m}(\mathbf{a}, \mathbf{d}) ; 1 \mu \mathrm{m}(\mathbf{b}$, $\mathbf{c}, \mathbf{e}, \mathbf{f})$

\section{Epithelial wound healing in cornea}

We then examined if lacking plakoglobin affects healing of an epithelial defect in cornea. Healing was similar between two genotypes of mice until $6 \mathrm{~h}$ post-epithelial debridement. Corneal epithelium-specific deletion of plakoglobin gene then retarded epithelial healing from $12 \mathrm{~h}$ post-wounding with a statistical significance at 12 and $30 \mathrm{~h}$ post-treatment as compared with a WT mouse (Fig. 7a, b).

\section{Cell proliferation assay}

We further examined if delayed epithelial healing was due to the impairment of cell proliferation in the epithelium, cell proliferation was analyzed in a different series of specimens by using BrdU-immunohistochemistry. The results showed no difference of the incidence of BrdU-labeled cells in the corneal epithelium between WT and mutant mice at 18 and $48 \mathrm{~h}$ post-wounding as well as in a healthy, uninjured, epithelium (Fig. 7c, d).

\section{Discussion}

Present study showed: (1) that the loss of plakoglobin altered the differentiation characteristics of the epithelium of a mouse cornea, (2) that it attenuated integrity of epithelial cells of the cornea and (3) that it impaired epithelial repair post-debridement without affecting cell proliferation.

\section{Alteration of the characteristics of corneal epithelium in the absence of plakoglobin}

Immunohistochemistry showed that keratin 14 was detected in basal cells of a WT corneal epithelium, while this type of keratin was more marked expressed not only in the basal cells but also cells in the suprabasal layers. Because keratin 14 is an established marker for a basal epithelial cell, present finding suggests that basal cell-type cells occupy the suprabasal layer.

Because plakoglobin is a component of cell-cell adhesion (adherence junction and desmosome) among epithelial cells, lacking this component might affect the distribution of epithelial cells in the stratified structure by loosening the cell-cell binding. It was to be examined if lacking plakoglobin might affect the vertical and/or horizontal cell arrangement in plakoglobin-null epithelium. For these two purposes we analyzed the cell number in a specific area of the corneal epithelium. The results showed that the number of basal cells decreased, while the total cell number was unchanged. This finding coincides with the results obtained by BrdU-immunohistochemistry; cell proliferation in the corneal epithelium was unaffected by the loss of plakoglobin. This finding suggested the loss of plakoglobin might have a negative impact on cell-cell adhesion and the basal cells might be pushed up toward the suprabasal layer due to the looser cell-cell connection.

Present study showed that lacking plakoglobin dramatically altered the localization of $\beta$-catenin in corneal epithelium. Plakoglobin and $\beta$-catenin share the role as a 
a
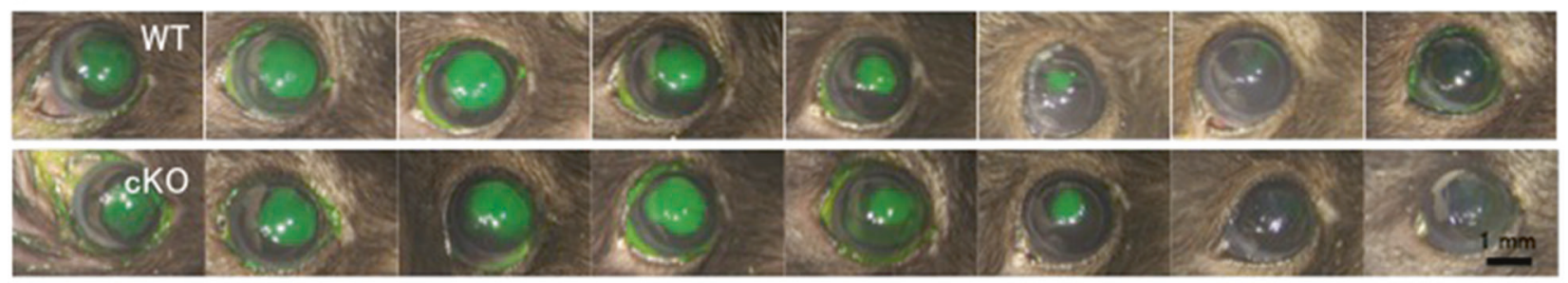

$0 \quad 6$

12

18

24

30

36

$42 \mathrm{hrs}$

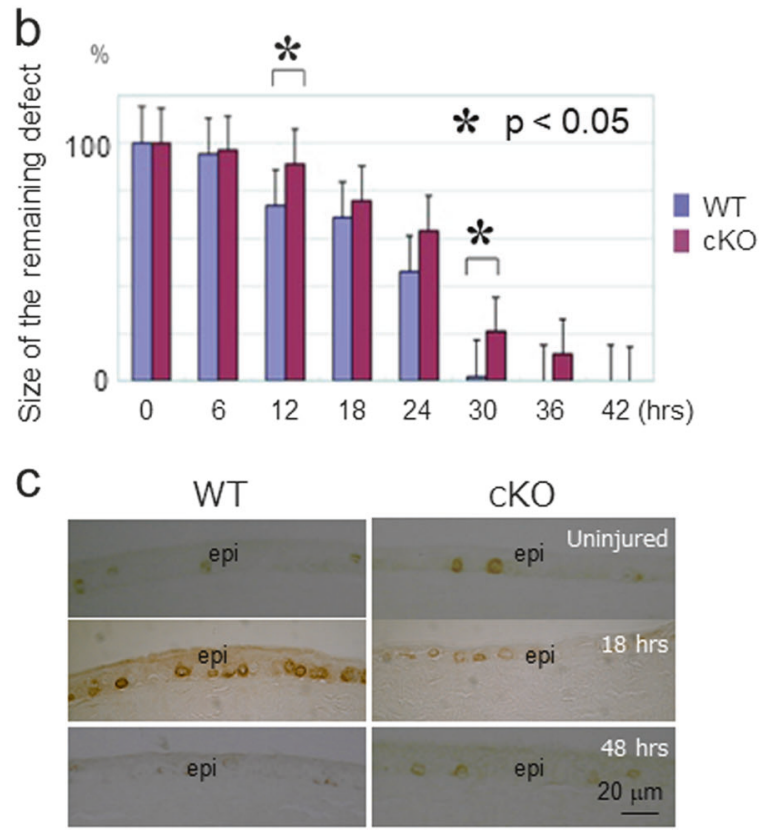

Fig. 7 Epithelial wound healing in cornea in a wild-type (WT) and a mutant mouse. a The round epithelial defect (stained with green fluorescein) produced in the center of the cornea is gradually recovered with the migrating remaining epithelium from outside the defect. The size of the remaining defect was evaluated with fluorescein staining of the defect at every $6 \mathrm{~h}$ until $42 \mathrm{~h}$ post-wounding. Bar, $1 \mathrm{~mm}$. b Healing is similar between two genotypes of mice until $6 \mathrm{~h}$ post-epithelial debridement. Corneal epithelium-specific deletion of plakoglobin gene then retards epithelial healing from $12 \mathrm{~h}$ post-wounding with a

component of desmosome. Thus, loss of plakoglobin could affect the signaling of $\beta$-catenin by its nuclear translocation. $\beta$-catenin was detected in the cytoplasm of the basal cells of the WT corneal epithelium. Its cytoplasmic localization was diminished by the loss of plakoglobin, while $\beta$-catenin was markedly condensed in cell-cell-border of epithelial cells throughout the layers of the $\mathrm{cKO}$ mouse. This finding could be explained by a notion that the loss of plakoglobin in adherence junction might be compensated by $\beta$-catenin [4]. However, there might be a possibility that alteration of intracellular distribution of $\beta$-catenin might affect its signal transduction in epithelial cells and behavior of the cells. For example, a literature reported that activation of $\beta$-catenin by Wnt5a was required for normal differentiation of keratinocytes in terms of keratin expression pattern [18]. $\beta$-catenin statistical significance at 12 and $30 \mathrm{~h}$ post-debridement. $(* p<0.05)$ c The incidence of proliferating cells in wild-type (WT) or plakoglobinnull (cKO) corneal epithelium (epi) was evaluated by using immunodetection of systemically administered bromo-deoxyuridine (BrdU) under healthy, uninjured, condition and at 18 and $48 \mathrm{~h}$ postdebridement. Bar, $20 \mu \mathrm{m}$. d There is no difference of the incidence of BrdU-labeled cells in the corneal epithelium between a WT and a mutant mouse at 18 and $48 \mathrm{~h}$ post-wounding as well as in a healthy, uninjured, epithelium

activated by Wnt5a promoted expression of keratin 1 and loricrin, markers for differentiation from a basal cell to a suprabasal cell of the stratified epithelium.

\section{Fragility of corneal epithelium in the absence of plakoglobin}

Present study also showed the critical role of plakoglobin in the maintenance of the integrity of mouse corneal epithelium by employing a mechanical intervention model to the tissue. Immunohistochemical examination showed that the loss of plakoglobin secondarily suppressed expression of epiplakin, an intermediate filament-associated component, in corneal epithelium. However, the present abnormal findings in plakoglobin-null corneal epithelium were not 
considered to be mainly attributable to the secondary reduction of epiplakin expression as the abnormalities in the absence of plakoglobin were not similar to those seen in epiplakin-null corneal epithelium. First, cell-cell adhesion among superficial cells was impaired in the absence of plakoglobin as observed by ultrastructural examination, while such loose adhesion was not observed epiplakin-null corneal epithelium in our previous study [11]. Although literatures reported that the loss of plakoglobin was compensated with $\beta$-catenin in desmosomes, loose cell-cell adhesion suggested that this compensation seemed insufficient in plakoglobin-null corneal epithelium. Second, a treatment of mechanical rubbing damaged structure of the corneal epithelium of a plakoglobin-null mouse. In mutant mice that lacked plakoglobin the rubbing treatment removed the superficial cells remaining suprabasal cells and basal cells relatively intact on the basement membrane. On the other hand, our previous study showed that the loss of epiplakin resulted in the disruption of the cytoplasm in the superficial and/or suprabasal cells following a treatment of mechanical rubbing in mice, suggesting that lacking epiplakin impaired cytoskeletal architecture in the cytoplasm. These findings suggested that disruption of epithelium integrity in the absence of plakoglobin did not seem to be attributable to the decreased expression of epiplakin.

\section{Impairment of epithelial healing in plakoglobin-null cornea}

Finally, present study herein showed that the loss of plakoglobin impaired healing of an epithelial defect in a mouse cornea. The major components of epithelial wound healing included cell migration and cell proliferation. In healing corneal epithelium cell migration first covered the defect, followed by the cell proliferation in the peripheral epithelium for cell supplementation and also proliferation in the monolayer epithelium in the defected area for the purpose of reestablishment of epithelial stratification. As for the role of plakoglobin in modulation of proliferation was under debate; literatures reported promotion or suppression of cell proliferation in each cell type $[9,19]$. Here there was no difference of cell proliferation in an uninjured cornea as well as healing cornea at 18 and $48 \mathrm{~h}$ post-debridement. It was reported that plakoglobin expression is related to modulation of cell migration via regulation of activation of signals through p38, Rho and Src [20, 21]. It was also reported that expression level of plakoglobin could affect cell death in vitro. However, in the current in vivo study TUNEL staining did not detect apoptotic cells in each layer of the epithelial cells (data not shown) [10, 22]. Therefore, we considered that the impairment of epithelial wound healing was mainly attributable to the suppression of cell migration by the loss of plakoglobin. Although the mechanism of cell migration retardation in the absence of plakoglobin needed to be elucidated, involvement of plakoglobin in cell migration promotion was reported in other cell types [19, 20,23].

In conclusion, the present study showed that plakoglobin is essential to the maintenance of the structure of the corneal epithelium and its wound healing. Although it had not been reported that patients with Naxos disease showed impairment of corneal epithelial integrity or tissue repair, current findings suggested an importance of ophthalmic examination of the disease.

Acknowledgements This work was supported by JSPS KAKENHI Grant Number JP15K10876 (to MK), NIH/NEI EY011845 \& Ohio Lions Eye Research Foundation (to WK), NIH R01-EY-23086 (to CYL), NIH/HL134599-01 (to WS).

\section{Compliance with ethical standards}

Conflict of interest The authors declare that they have no conflict of interest.

\section{References}

1. Ziaei M, Greene C, Green CR. Wound healing in the eye: therapeutic prospects. Adv Drug Deliv Rev. 2018 Jan 20. pii: S0169409X(18)30006-1. https://doi.org/10.1016/j.addr.2018.01.006

2. Bukowiecki A, Hos D, Cursiefen C, et al. Wound-healing studies in cornea and skin: parallels, differences and opportunities. Int $\mathrm{J}$ Mol Sci. 2017;18:e1257.

3. Liu CY, Kao WW. Corneal epithelial wound healing. Prog Mol Biol Transl Sci. 2015;134:61-71.

4. Peifer M, McCrea PD, Green KJ, et al. The vertebrate adhesive junction proteins beta-catenin and plakoglobin and the Drosophila segment polarity gene armadillo form a multigene family with similar properties. J Cell Biol. 1992;118:681-91.

5. Jani ELewis, Wahl JamesK III, Sass KristinM, et al. Cross-talk between adherens junctions and desmosomes depends on plakoglobin. J Cell Biol. 1997;136:919-34.

6. Protonotarios N, Tsatsopoulou A. Naxos disease: Cardiocutaneous syndrome due to cell adhesion defect. Orphanet J Rare Dis. 2006;1:4.

7. Asimaki A, Syrris P, Wichter T, Matthias P, et al. A novel dominant mutation in plakoglobin causes arrhythmogenic right ventricular cardiomyopathy. Am J Hum Genet. 2007;81:964-73.

8. Aktary Z, Alaee M, Pasdar M. Beyond cell-cell adhesion: plakoglobin and the regulation of tumorigenesis and metastasis. Oncotarget . 2017;19:32270-91.

9. Bierkamp C, Mclaughlin KJ, Schwarz H, et al. Embryonic heart and skin defects in mice lacking plakoglobin. Dev Biol. 1996;180:780-5.

10. Li D, Zhang W, Liu Y, et al. Lack of plakoglobin in epidermis leads to keratoderma. J Biol Chem. 2012;287:10435-43.

11. Kokado M, Okada Y, Goto M, et al. Increased fragility, impaired differentiation, and acceleration of migration of corneal epithelium of epiplakin-null mice. Invest Ophthalmol Vis Sci. 2013;54:3780-9.

12. Li D, Liu Y, Maruyama M, et al. Restrictive loss of plakoglobin in cardiomyocytes leads to arrhythmogenic cardiomyopathy. Hum Mol Genet. 2011;20:4582-96.

13. Hayashi Y, Call MK, Liu CY, et al. Monoallelic expression of Krt12 gene during corneal-type epithelium differentiation of limbal stem cells. Invest Ophthalmol Vis Sci. 2010;51:4562-8. 
14. Okada Y, Ikeda K, Yamanaka O, et al. TNFalpha suppression of corneal epithelium migration. Mol Vis. 2007;13:1428-35.

15. Saika S, Ikeda K, Yamanaka O, et al. Expression of Smad7 in mouse eyes accelerates healing of corneal tissue after exposure to alkali. Am J Pathol. 2005;166:1405-18.

16. Kao WW, Liu CY, Converse RL, et al. Keratin 12-deficient mice have fragile corneal epithelia. Invest Ophthalmol Vis Sci. 1996;37:2572-84.

17. Saika S, Okada Y, Miyamoto T, et al. Role of p38 MAP kinase in regulation of cell migration and proliferation in healing corneal epithelium. Invest Ophthalmol Vis Sci. 2004;45:100-9.

18. Popp Tanja, Steinritz Dirk, Breit Andreas, Deppe Janina, et al. Wnt $5 \mathrm{a} / \beta$-Catenin signaling drives calcium-induced differentiation of human primary keratinocytes. J Invest Dermatol. 2014; 134:2183-91.
19. Aktary Z, Pasdar M. Plakoglobin represses SATB1 expression and decreases in vitro proliferation, migration and invasion. PLoS One. 2013;8:e78388.

20. Spindler V, Dehner C, Hübner S, et al. Plakoglobin but not desmoplakin regulates keratinocyte cohesion via modulation of p38MAPK signaling. J Invest Dermatol. 2014;134:1655-64.

21. Todorović V, Desai BV, Patterson MJ, et al. Plakoglobin regulates cell motility through Rho- and fibronectin-dependent Src signaling. J Cell Sci. 2010;123:3576-86.

22. Wei Q, Hariharan V, Huang H. Cell-cell contact preserves cell viability via plakoglobin. PLoS One. 2011;6:e27064.

23. Sechler M, Borowicz S, Van Scoyk M, et al. Novel role for $\gamma$ catenin in the regulation of cancer cell migration via the induction of hepatocyte growth factor activator inhibitor type 1 (HAI-1). J Biol Chem. 2015;290:15610-20. 